\title{
REGULARITY OF BANACH ALGEBRAS GENERATED BY ANALYTIC SEMIGROUPS SATISFYING SOME GROWTH CONDITIONS
}

\author{
J. ESTERLE AND J. E. GALÉ 1
}

ABstract. We show that if a commutative complex Banach algebra $A$ is generated by a nonzero analytic semigroup $\left(a^{t}\right)_{\operatorname{Re} t>0}$ satisfying

$$
\int_{-\infty}^{+\infty} \frac{\log ^{+}\left\|a^{1+i t}\right\|}{1+t^{2}} d t<+\infty
$$

then $A$ is regular in Shilov's sense.

1. Introduction. Recall that a commutative (nonradical) complex Banach algebra $A$ is said to be regular in Shilov's sense, or regular for short, if, given $\alpha_{0} \in \hat{A}$ and a neighbourhood $\mathcal{U}$ of $\alpha_{0}$ in $\hat{A}$ there exists $b \in A$ with $\alpha_{0}(b) \neq 0$ and $\alpha(b)=0$ for every $\alpha \notin \mathcal{U}$. (Here we denote by $\hat{A}$ the carrier space of $A$ equipped with the Gelfand topology.)

The classical proofs of the Wiener tauberian theorem for the convolution algebra $L^{1}(R)$ use the fact that $L^{1}(R)$ is regular. The Wiener tauberian theorem extends to the weighted algebras $L^{1}\left(R, e^{\phi(t)}\right)$ where $\phi$ is a nonnegative, measurable function on $R$ satisfying $\phi(s+t) \leq \phi(s)+\phi(t)(s, t \in R)$ and

$$
\int_{-\infty}^{+\infty} \frac{\phi(t)}{1+t^{2}} d t<+\infty
$$

(the first condition just ensures that $L^{1}\left(R, e^{\phi(t)}\right)$ is stable under convolutions), and Beurling's original proof of this result $[\mathbf{1}]$ is also based upon the regularity of $L^{1}\left(R, e^{\phi(t)}\right)$. There are some noncommutative extensions of Wiener's tauberian theorem to the group algebras $L^{1}(G)$ where $G$ is, for example, a nilpotent Lie group. In that case it is true that if $I$ is a two-sided ideal of $L^{1}(G)$ such that $I \neq L^{1}(G)$ then the quotient algebra $L^{1}(G) / I$ is not radical (in other terms $L^{1}(G)$ possesses the "weak Wiener property" in the sense of Leptin $[\mathbf{7}])$. This fact is proved by Hulanicki in [5] using the regularity of the commutative subalgebra of $L^{1}(G)$ generated by the "heat semigroup" (see [7] for a discussion of noncommutative one-sided or two-sided extensions of the tauberian theorem).

In [4] the first author gives a proof of the Wiener tauberian theorem for $L^{1}(R)$ based upon an application of the Ahlfors-Heins theorem [2, Theorem 7-2-6] to the heat semigroup. Dales and Hayman give in [3] a proof of the tauberian theorem for some Beurling algebras which also involves the growth on vertical lines of the heat

Received by the editors December 12, 1982.

1980 Mathematics Subject Classification. Primary 46J30.

Key words and phrases. Regular Banach algebra, analytic semigroup.

${ }^{1}$ The research of the second author was supported by a Beca del Plan de Formacion del Personal Investigador, Spain, in the University of Bordeaux, France. 
semigroup and some more sophisticated results in the theory of analytic functions. Hulanicki [6] observed that the argument of [4] applied to the heat semigroup on a nilpotent Lie group $G$ gives also a proof of the weak Wiener property for $L^{1}(G)$. In fact the method used in [4] can be applied to Banach algebras $A$ which possess an analytic semigroup $\left(a^{t}\right)_{\operatorname{Re} t>0}$ such that $\left[a^{t} A\right]^{-}=A(\operatorname{Re} t>0)$,

$$
\int_{-\infty}^{+\infty} \frac{\log ^{+}\left\|a^{1+i y}\right\|}{1+y^{2}} d y<+\infty .
$$

As shown in [8, Lemma 5-7] such an analytic semigroup is of exponential type in the half-plane $\operatorname{Re} z \geq 3$, and the Ahlfors-Heins theorem shows then that every proper two-sided ideal of $A$ is contained in a primitive ideal of $A$ [8, Theorem 5-6]. This use of the Ahlfors-Heins theorem avoids any reference to regularity.

We show here in the other direction that the above growth condition implies that a large commutative subalgebra of $A$ is regular. More precisely we have the following theorem.

THEOREM 1. Let $\left(a^{t}\right)_{\operatorname{Re} t>0}$ be a nonzero analytic semigroup in a Banach algebra $A$. If

$$
\int_{-\infty}^{\infty} \frac{\log ^{+}\left\|a^{1+i t}\right\|}{1+t^{2}} d t<+\infty
$$

then the closed commutative subalgebra $B$ of $A$ generated by $\left\{a^{t}\right\}_{\operatorname{Re} t>0}$ is regular.

2. Proof of the theorem. Note that Theorem $5-6$ of $[8]$ shows that $B$ is not radical. We will need two lemmas.

LEMMA 1. Put $\omega(t)=\sup \left(1,\left\|a^{2+i t}\right\|\right)(t \in R)$. Then for every $y_{0} \in R$ and every $\eta>0$ there exists an element $f$ of the Banach space $L^{1}(R, \omega)$ whose Fourier transform $\hat{f}$ satisfies $\hat{f}\left(y_{0}\right)=1, \hat{f}(y)=0$ if $\left|y-y_{0}\right| \geq \eta$.

Proof. Put $\omega_{0}(t)=\sup \left(1,\left\|a^{1+i t}\right\|\right)(t \in R)$. Note that $L^{1}\left(R, \omega_{0}\right)$ and $L^{1}(R, \omega)$ are contained in $L^{1}(R)$, so that the convolution product $g_{1} * g_{2}$ is well defined for every $g_{1}, g_{2} \in L^{1}\left(R, \omega_{0}\right)$ and the Fourier transform $\hat{f}$ of $f$ is well defined for every $f \in L^{1}(R, \omega) \cup L^{1}\left(R, \omega_{0}\right)$. As $\omega(s+t) \leq \omega_{0}(s) \omega_{0}(t)(s, t \in R)$, it follows from a standard application of Fubini's theorem that $g_{1} * g_{2} \in L^{1}(R, \omega)$ for every $g_{1}, g_{2} \in L^{1}\left(R, \omega_{0}\right)$.

We have $\omega_{0}(t)=e^{\log ^{+}\left\|a^{1+i t}\right\|}(t \in R)$ where

$$
\int_{-\infty}^{+\infty} \frac{\log ^{+}\left\|a^{1+i t}\right\|^{2}}{1+t^{2}} d t<+\infty
$$

A classical argument recalled in $[3]$ shows then that there exists $g_{1} \in L^{1}\left(R, \omega_{0}\right)$ such that $\hat{g}_{1}\left(y_{0}\right)=1, \hat{g}_{1}(y)=0$ for every $y \in\left(-\infty, y_{0}-\eta\right]$ and $g_{2} \in L^{1}\left(R, \omega_{0}\right)$ such that $\hat{g}_{2}\left(y_{0}\right)=1, \hat{g}_{2}(y)=0$ for every $y \in\left[y_{0}+\eta, \infty\right)$. Then $f=g_{1} * g_{2}$ belongs to $L^{1}(R, \omega)$ and satisfies the conditions of the lemma.

LEMMA 2. For every character $\alpha$ of $B$ there exists a unique real number $\tilde{\alpha}$ such that $\alpha\left(a^{t}\right)=\exp (i \tilde{\alpha} t)(\operatorname{Re} t>0)$.

Proof. The map $\theta: t \mapsto \alpha\left(a^{t}\right)$ is analytic, and $\theta\left(t+t^{\prime}\right)=\theta(t) \theta\left(t^{\prime}\right)$ if $\operatorname{Re} t>$ $0, \operatorname{Re} t^{\prime}>0$. So there exists $\tilde{\alpha} \in \mathbf{C}$ such that $\alpha\left(a^{t}\right)=\exp (i \tilde{\alpha} t)(\operatorname{Re} t>0)$. Put 
$\tilde{\alpha}=\lambda+i \mu$ where $\lambda$ and $\mu$ are real. Then $\left|\alpha\left(a^{1+i t}\right)\right|=\exp (\lambda-t \mu)(t \in R)$ and as

$$
\int_{-\infty}^{+\infty} \frac{\log ^{+}\left|\alpha\left(a^{1+i t}\right)\right|}{1+t^{2}} d t<+\infty
$$

we must have $\mu=0$. So $\tilde{\alpha}$ is a real number. If another real number $\delta$ satisfies the condition $\alpha\left(a^{t}\right)=\exp (i \delta t)(\operatorname{Re} t>0)$ then $(\tilde{\alpha}-\delta) / 2 \pi t \in Z$ for every $t$, so that $\tilde{\alpha}=\delta$ and $\alpha$ is unique. This proves the lemma.

We can now prove the theorem. Let $\alpha_{0} \in \hat{B}$ and let $U$ be a neighborhood of $\alpha_{0}$ in $\hat{B}$. Note that if a sequence $\left(\beta_{n}\right)$ of elements of $\hat{B}$ satisfies

$$
\left|\tilde{\beta}_{n}-\tilde{\alpha}_{0}\right| \underset{n \rightarrow \infty}{\longrightarrow} 0
$$

then $\alpha_{0}\left(a^{t}\right)=\lim _{n \rightarrow \infty} \beta_{n}\left(a^{t}\right)$ for every $t$, so that $\left(\beta_{n}\right)$ converges towards $\alpha_{n}$ in $\hat{B}$. We thus see that there exists $\eta>0$ such that $\left|\tilde{\alpha}_{0}-\tilde{\beta}\right| \geq \eta$ for every $\beta \notin \mathcal{U}$.

It follows from Lemma 1 that there exists $f \in L^{1}(R, \omega)$ such that

$$
\int_{-\infty}^{+\infty} f(t) e^{i t \tilde{\alpha}_{0}} d t=1 \text { and } \int_{-\infty}^{+\infty} f(t) e^{i t \lambda} d t=0
$$

if $\left|\lambda-\tilde{\alpha}_{0}\right| \geq \eta$. Then

$$
\int_{-\infty}^{+\infty}|f(t)|\left\|a^{2+i t}\right\| d t<+\infty
$$

and the Bochner integral

$$
\int_{-\infty}^{+\infty} f(t) a^{2+i t} d t
$$

defines an element $b$ of $B$.,

As Bochner integrals commute with continuous linear forms, we have

$$
\begin{aligned}
\alpha_{0}(b) & =\int_{-\infty}^{+\infty} f(t) \alpha_{0}\left(a^{2+i t}\right) d t \\
& =e^{2 \tilde{\alpha}_{0}} \int_{-\infty}^{+\infty} f(t) e^{i t \tilde{\alpha}_{0}} d t=e^{2 \tilde{\alpha}_{0}} \neq 0 .
\end{aligned}
$$

We see similarly that $\beta(b)=0$ for every $\beta \neq \mathcal{U}$, as $\left|\tilde{\beta}-\tilde{\alpha}_{0}\right| \geq \eta$. This concludes the proof of the theorem.

REMARK. If a semigroup $\left(a^{t}\right)_{\operatorname{Re} t>0}$ in a Banach algebra $A$ satisfies the growth condition of Theorem 1 then $a^{t} \in \operatorname{Sp}\left(a^{n}\right)_{n \in N}$ for every $t$, and the closed subalgebra of $A$ generated by the semigroup equals the closed subalgebra generated by $a^{1}$. To see this note that

$$
\int_{-\infty}^{+\infty} \frac{\log ^{+}\left|l\left(a^{1+i t}\right)\right|}{1+t^{2}} d t<+\infty
$$

for every continuous linear form $l$ on $A$. As the function $t \mapsto l\left(a^{t}\right)$ is analytic for Re $t \geq 1$ we must have $l\left(a^{t}\right)=0$ for every $t$ if $l\left(a^{n}\right)=0$ for every $n \geq 1[2$, Theorem $9-3-8]$. 


\section{REFERENCES}

1. A. Beurling, Sur les intégrales de Fourier absolument convergentes et leur application à une transforma tion fonctionnelle, Neuvième Congrès Math. Scandinaves (Helsinki, 1938), Tryekeri, Helsinki, 1939, pp. 199-210.

2. R. P. Boas, Jr., Entire functions, Academic Press, New York, 1954.

3. H. G. Dales and W. K. Hayman, Esterle's proof of the tauberian theorem for Beurling algebras, Ann. Inst. Fourier (Grenoble) 31 (1981), 141-150.

4. J. Esterle, A complex-variable proof of the Wiener tauberian theorem, Ann. Inst. Fourier (Grenoble) 30 (1980), 91-96.

5. A. Hulanicki, Subalgebra of $L^{1}(G)$ associated with Laplacian on a Lie group, Colloq. Math. 31 (1974), 259-287.

6.

7. H. Leptin, Ideal theory in group algebras of locally compact groups, Invent. Math. 31 (1976), 259278.

8. A. M. Sinclair, Continuous semigroups in Banach algebras, London Math. Soc. Lecture Notes, vol. 63, Cambridge Univ. Press, 1982.

LABORATOIRE ASSOCIÉ AU C.N.R.S., U.E.R. DE MATHÉMATIQUES ET INFORMATIQUE, Université de Bordeaux I, 351, COURS de la Libération, 33405-TAlence, France

departamento de Teoría de Funciones, facultad de Ciencias, Universidad DE ZARAGOZA, ZARAGOZA, SPAIN 